\title{
Association of VDR gene Taql polymorphism with the susceptibility to prostate cancer in Asian population evaluated by an updated systematic meta-analysis
}

This article was published in the following Dove Press journal:

OncoTargets and Therapy

\author{
Liangliang Chen* \\ Junjun Wei* \\ Shuwei Zhang \\ Zhongguan Lou \\ Xue Wang \\ Yu Ren \\ Honggang Qi \\ Zhenhua Xie \\ Yirun Chen \\ Feng Chen \\ Qihang Wu \\ Xiaoxiao Fan \\ Honglei Xu \\ Shuaishuai Huang \\ Guobin Weng
}

Department of Urologic Surgery, Ningbo Urology and Nephrology Hospital, Urology and Nephrology Institute of Ningbo University, Ningbo, China

*These authors contributed equally to this work

Correspondence: Shuaishuai Huang; Guobin Weng

Ningbo Urology and Nephrology Hospital, Urology and Nephrology Institute of Ningbo University,

998 North Qianhe Road, Yinzhou,

Ningbo, Zhejiang 315000, China

Tel +865745 5662926

Email ss.huang@aliyun.com;

ddwgbin@aliyun.com
Background: The vitamin D receptor (VDR) plays a key role in vitamin-mediated signaling pathway. Emerging evidence has suggested that the VDR polymorphism may contribute to the risk of prostate cancer $(\mathrm{PCa})$. However, the existing results are not conclusive in Asian population.

Methods: We aim to evaluate the potential role of VDR polymorphisms on PCa of Asian population. PubMed, Scopus, Embase, Web of Science, Chinese National Knowledge Infrastructure, Wang Fang Data, and VIP Periodical were retrieved, and eligible studies (casecontrol or cohort study) meeting the inclusion criteria were evaluated through an updated meta-analysis using Stata13.0 software.

Results: A total of 1,363 cases and 2,101 controls obtained from 13 eligible publications were eventually included in this meta-analysis. Our results show that a significant association of VDR taq1 polymorphism with PCa risk, especially in the Japanese population. In the clinical stagestratified analysis, the pooled results revealed no significant difference in genetic polymorphisms between the local stage and control groups, whereas there was increased frequency of $\mathrm{T}$ allele and TT genotype in the advanced tumor stage group compared with local tumor stage or control groups. Similarly, no significant difference was seen in Gleason $<7$ and control groups, but the $\mathrm{T}$ allele and TT genotype were significantly higher in the Gleason $\geq 7$ group compared with Gleason $<7$ or control groups.

Conclusion: The VDR TaqI polymorphism might be associated with PCa risk in Asian population, especially in the Japanese population. Also, PCa patients carrying the T allele or TT genotype were more likely to progress to advanced stage. These results suggest that VDR TaqI polymorphisms may be potential diagnostic biomarkers for PCa susceptibility.

Keywords: vitamin D receptor, polymorphism, prostate cancer, clinical stage, meta-analysis

\section{Background}

Prostate cancer ( $\mathrm{PCa}$ ), is one of the most common malignant tumors and the second leading cause of male mortality worldwide. In the USA, a total number of 180,890 new cases and 26,120 deaths occurred in 2016. ${ }^{1}$ Although the pathogenesis of PCa is not clearly elucidated, age, ethnicity, and genetic factors are believed to contribute to the occurrence of $\mathrm{PCa} .^{2}$

Some reports suggested that low levels of vitamin $\mathrm{D}$ in serum might be a risk factor for PCa. ${ }^{3}$ The hormonally active metabolite of vitamin D, 1,25(OH)2D3 (calcitriol), shows an anti-proliferative effect on many PCa cell lines. ${ }^{2,45}$ The transformation of 
vitamin $\mathrm{D}$ to calcitriol requires the binding of vitamin $\mathrm{D}$ receptor (VDR) and the complex of vitamin D-VDR-retinoid $\mathrm{X}$ receptor complex to regulate the cell proliferation, cell cycle, and apoptosis-related genes. ${ }^{6}$ High levels of VDR mediate the activation of vitamin $\mathrm{D}$ as active vitamin $\mathrm{D}[1,25$ $(\mathrm{OH}) 2 \mathrm{D} 3]$, which could inhibit the proliferation of normal prostate tissue and PCa epithelial cells, thus inhibiting the growth of PCa cells. ${ }^{4}$ This discovery suggested that the variation in VDR gene affects the prognosis or risk of PCa. TaqI, one of the most extensive single-nucleotide polymorphisms located in exon 9 of VDR gene might alter VDR mRNA levels. This is through regulation of mRNA stability and the protein translation efficiency, which may be responsible for the reduction in VDR level. ${ }^{7-9}$

Currently, existing evidence is insufficient to draw robust conclusions. ${ }^{10,11}$ Three previous meta-analyses have identified that there are no positive results in VDR TaqI polymorphism and PCa development, ${ }^{7,12,13}$ while study of Fei et al showed an inversed result in Asian population. Regrettably, no further subgroup analysis was conducted in different countries. ${ }^{14}$ The inclusion of population with benign prostatic hyperplasia (BPH) as control group is noted, and a relation of VDR TaqI polymorphism to BPH risk was also reported in some other studies. Our study considered the subjects as 1) healthy group, 2) BPH group, and 3) case group. Four new studies have provided additional data. ${ }^{15-18} \mathrm{We}$, therefore, conducted an updated meta-analysis to investigate the role of VDR TaqI polymorphism and PCa risk.

\section{Methods}

\section{Literature and research strategy}

PubMed, Scopus, Embase, Web of Science, Chinese National Knowledge Infrastructure, Wang Fang data, and VIP Periodical were searched using the following terms: ("Polymorphisms") and ("vitamin D receptor" OR "vitamin D3 receptor" OR “1,25- dihydroxyvitamin D3 receptor” OR "calcitriol receptor" OR "VDR"”) and ("PCa" OR "prostate neoplasm" OR "prostate tumor" OR "prostate carcinoma" OR "prostatic neoplasm"). Articles related to the association of VDR Taq1 polymorphisms and Asian PCa risk were obtained. Various combinations of the terms were also used in other databases. Literature search data were last updated on October 10, 2016. No limitations were attached.

\section{Inclusion and exclusion criteria}

In this meta-analysis, it was a must for selected studies to meet the following criteria:

(1) Studies that performed the associations of VDR Taq1 genetic polymorphisms and $\mathrm{PCa}$;
(2) Study objects belonged to the Asian population;

(3) Local stage PCa was defined as clinical and pathological stage of T1-T2N0M0 or stage A-B; advanced PCa was defined as tumor invasion outside the prostate envelope or cancer metastasis to the lymph nodes or other organ tissue, clinical pathological stage for T3-T4NxMx or TxN1 or M1; stage C and D;

(4) Gleason stage: low grade (Gleason $<7$ ) and high grade (Gleason $\geq 7$ );

(5) The odds ratios (ORs) with $95 \%$ CIs could be calculated or provided through detailed genotype data in casecontrol, cohort groups, and GWAS studies.

Exclusion criteria:

(1) No detailed genotype data or the raw data were available for retrieval;

(2) Comments, review articles, editorials, and other metaanalysis;

(3) For multiple publications from the same population/area, only the largest sample was included.

\section{Data extraction and synthesis}

Data of eligible studies were extracted independently by 2 independent reviewers, including the following contents: name of first author, year of publication, country of origin, characteristics of cases and controls, number of cases and controls, sources of controls, pathologic diagnosis, clinical pathologic staging, Gleason score, and Newcastle-Ottawa Scale (NOS). Extracted data with discrepancies in identification were discussed to reach a consensus. A third investigator assisted by adjudicating the disagreements if dissent still existed.

\section{Evaluation of the eligible studies' quality}

We used the NOS to evaluate each in accordance with the requirements of article quality, NOS score a total of 9 points, while each indicator score 1 point. If the score $>4$ points, it was considered to be a high quality article. ${ }^{19}$

\section{Statistical analysis}

We evaluated the pooled OR and 95\% CI to indicate the relationship between VDR gene Taq1 polymorphism and susceptibility to $\mathrm{PCa}$ by $Z$-test, with $P<0.05$ considering as statistically significant. Statistical heterogeneity among studies was carried out by the $Q$ statistic (significance level of $P<0.1)$ and $I^{2}$ statistic $(>50 \%$ as evidence of significant inconsistency). ${ }^{20,21}$ The $Q$-test and $I^{2}$ statistic were applied to determine the effect models according to heterogeneity. When $P$-value of heterogeneity $\left(P_{H}\right)$ was no $>0.1\left(P_{H} \leq 0.1\right)$, random effects model was used, and when $P_{H}$ was $>0.1$, 
fixed effects model was used. ${ }^{22}$ Sensitivity analysis was also tested by removing 1 study at a time, to evaluate the effect of removal and effect of size of each study on the homogeneity of the whole. In addition, subgroup analyses were stratified by countries, cancer stages, and Gleason stages. Publication bias was investigated with Begg's funnel plots and further assessed by the Egger's regression test. ${ }^{23,24}$ When an asymmetric plot was shown or Egger's test was $P<0.05$, we considered it as a significant publication bias. Besides, Hardy-Weinberg equilibrium (HWE) was implemented to identify the effective records in our study. All analyses were performed with Stata 13.0 software (StataCorp, College Station, TX, USA). A 2-tailed $P \leq 0.05$ was regarded as significant, except for specified conditions, for which a certain $P$-value was declared.

\section{Results}

\section{Study selection and characteristics}

A flow diagram of literature research and process is shown in Figure 1. Each study was retrieved and carefully reviewed according to the inclusive and exclusive criteria. Ultimately, a total of 13 articles reporting the relationship between Taq1 polymorphisms and PCa risk in Asian population were identified through both English and Chinese databases, ${ }^{15-18,25-33}$

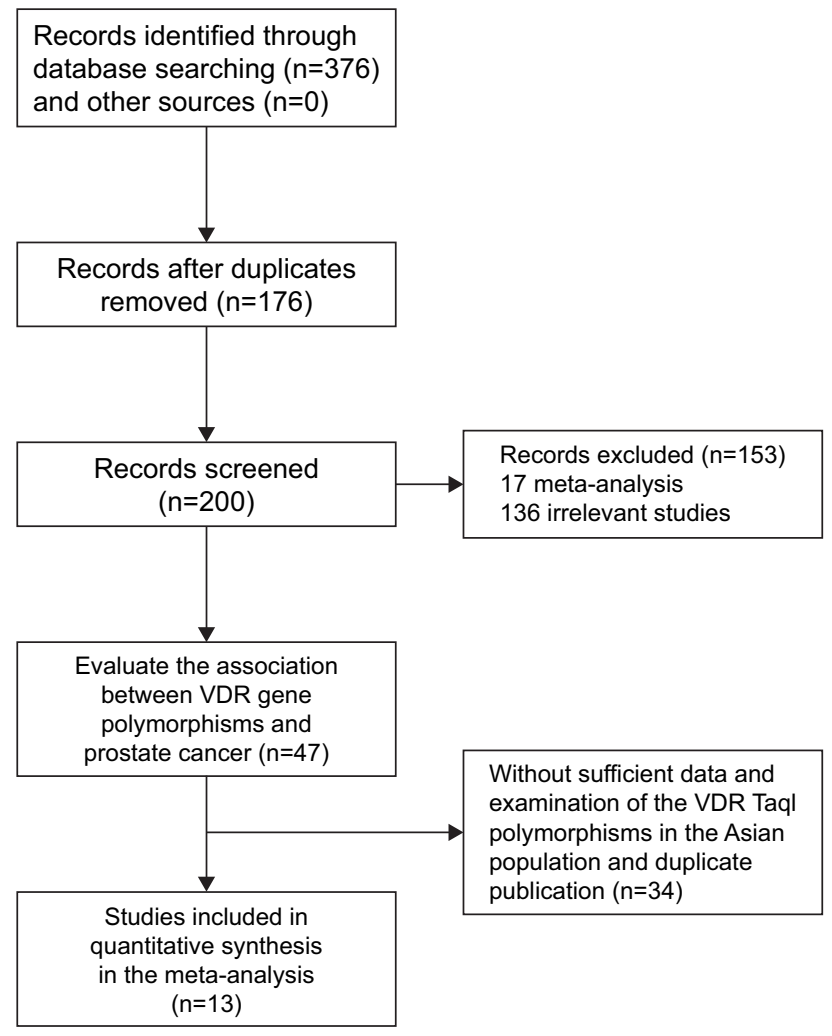

Figure I Study flowchart for the process of selecting the final 13 studies. Abbreviation: VDR, vitamin D receptor. including 1,363 cases and 2,101 controls (including healthy and BPH). Quality of each included article was evaluated by NOS, and each had a score of $\geq 5$, meaning a high quality, as listed in Table 1. In addition, we found that 3 studies did not meet HWE. ${ }^{15,25,33}$ We found no significant changes of the pooled results after excluding each article once.

\section{Association of VDR gene Taq I polymorphism with PCa risk in Asian population}

A total of 13 eligible articles, including 1,363 cases and 2,101 controls (including non-BPH and $\mathrm{BPH}$ ) were analyzed the association between Taq1 polymorphism of VDR and PCa risk in Asian population. As is presented in Table 2, TaqI genetic polymorphism was significantly associated with the risk of $\mathrm{PCa}$ ( $\mathrm{T}$ vs t: OR $[95 \% \mathrm{CI}]=1.23$ [1.05-1.44], $P=0.010$; TT vs tt: OR [95\% CI] $=1.99$ [1.20-3.30], $P=0.008$; [TT/Tt] vs tt: OR [95\% CI]=2.03 [1.24-3.34], $P=0.005)$; (Figure 2A, D and G). Begg's test revealed no publication bias (Figure 2B, E and $\mathrm{H}$ ), and the sensitivity analysis was stable (Figure 2C, F and I).

Previous study suggested that the inclusion of population with $\mathrm{BPH}$ as control group may bias the pooled results to a varying degree. ${ }^{14}$ In order to draw attention to this point, we divided the control into 2 groups, BPH and non-BPH control. As a result, a marked association was performed with Taq1 polymorphism and $\mathrm{PCa}$ risk in the case vs non-BPH subgroup (T vs t: OR [95\% CI] $=1.54$ [1.20-1.98], $P=0.001$; TT vs Tt: OR [95\% CI] $=1.41$ [1.06-1.87], $P=0.017$; TT vs tt: OR $[95 \% \mathrm{CI}]=3.53[1.40-8.94], P=0.008$; TT vs $[\mathrm{Tt} / \mathrm{tt}]$ : OR $[95 \% \mathrm{CI}]=1.51[1.15-1.98], P=0.003$; [TT/Tt $]$ vs tt: OR [95\% CI] $=3.15$ [1.25-7.94], $P=0.015$; Figure 3A, D, G, J and $\mathrm{M}$ ), while no significant difference was found between BPH and non-BPH subgroup. The results revealed that VDR Taq1 polymorphism was not associated with BPH. Begg's test revealed no publication bias, and the sensitivity analysis was stable (Table 2 and Figure 3).

\section{Association of VDR gene Taql polymorphism with PCa risk in different Asian countries}

We performed a subgroup analysis according to different Asian countries. As shown in Figure 4 and Table 3, the pooled results indicated that VDR TaqI genetic polymorphism was closely linked with PCa risk in Japanese population (T vs t: OR [95\% $\mathrm{CI}]=1.23$ [1.0-1.51], $P=0.049$; TT vs tt: OR [95\% CI] $=2.04$ [1.00-4.16], $P=0.048)$, while no association was shown in Chinese (4 studies), Pakistan (1 study), India (1 study), and Thailand (1 study) population. Begg's test revealed no 


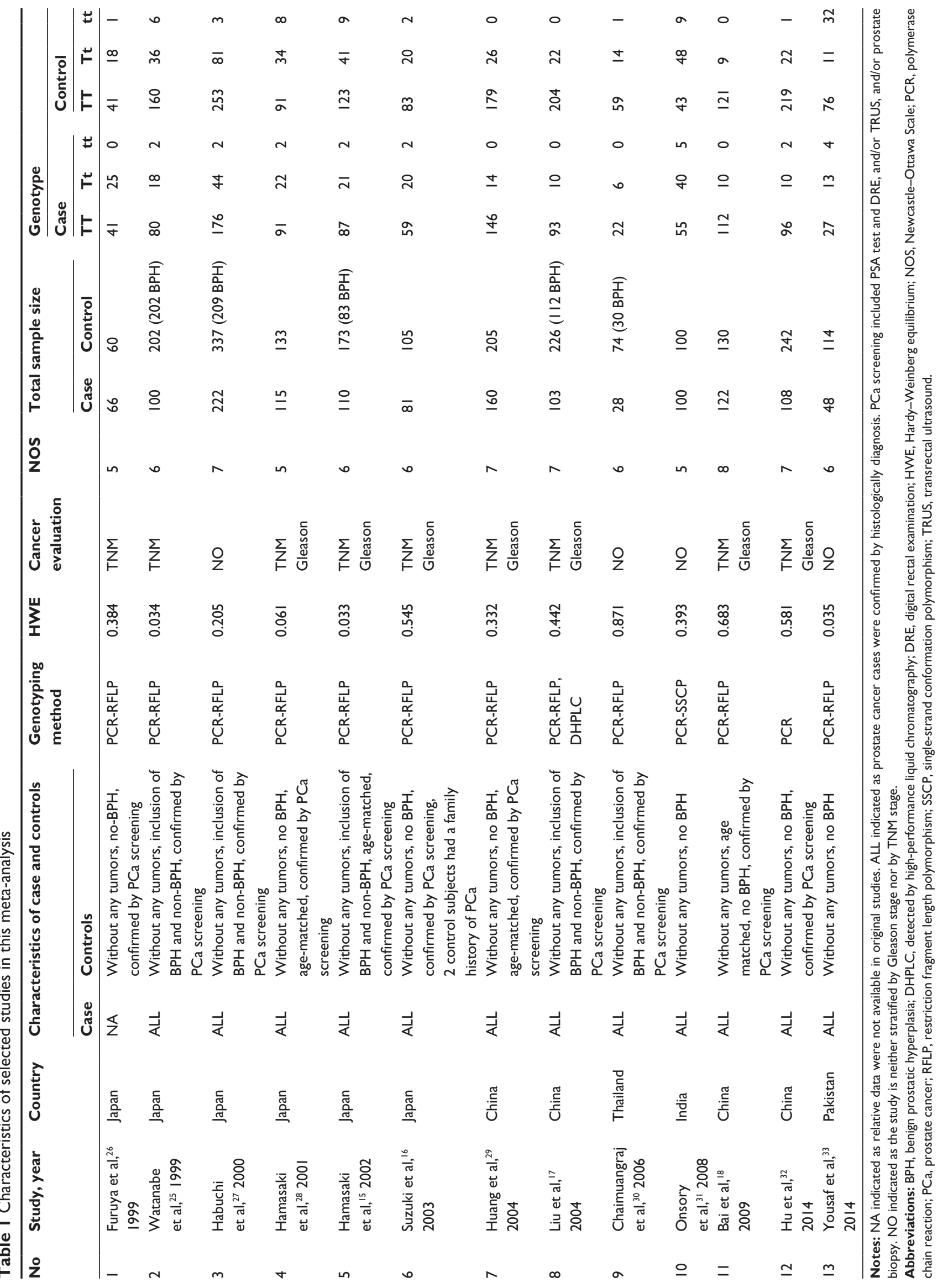


Table 2 Meta-analysis of the association of VDR gene Taq I polymorphism with PCa risk with different groups in Asian population

\begin{tabular}{|c|c|c|c|c|c|c|c|c|c|}
\hline \multirow[t]{2}{*}{ Gene } & \multirow{2}{*}{$\frac{\text { Studies }}{\mathbf{N}}$} & \multicolumn{3}{|c|}{ Test for overall effect } & \multicolumn{2}{|c|}{ Heterogeneity } & \multirow[t]{2}{*}{$M$} & \multicolumn{2}{|c|}{ Publication bias } \\
\hline & & OR $(95 \% \mathrm{Cl})$ & Z-score & $P$-value & $I^{2}$ & $P$-value & & $\begin{array}{l}\text { Begg's } \\
\text { test }\end{array}$ & $\begin{array}{l}\text { Egger's } \\
\text { test }\end{array}$ \\
\hline \multicolumn{10}{|c|}{ Case vs all control } \\
\hline T vs $t$ & 13 & $1.23(1.05-1.44)$ & 2.56 & 0.010 & $0 \%$ & 0.460 & $\mathrm{~F}$ & 0.127 & 0.092 \\
\hline TT vs Tt & 13 & $1.09(0.9|-| .31)$ & 0.96 & 0.330 & $23.4 \%$ & 0.207 & $\mathrm{~F}$ & 0.024 & 0.024 \\
\hline TT vs tt & 13 & $1.99(1.20-3.30)$ & 2.67 & 0.008 & $0 \%$ & 0.681 & $\mathrm{~F}$ & 0.049 & 0.126 \\
\hline TT vs $(T t / t t)$ & 13 & $1.17(0.98-1.40)$ & 1.77 & 0.076 & $0 \%$ & $0.58 \mathrm{I}$ & $\mathrm{F}$ & 0.100 & 0.057 \\
\hline$(\mathrm{TT} / \mathrm{Tt}) \mathrm{vs} \mathrm{tt}$ & 13 & $2.03(1.24-3.34)$ & 2.80 & 0.005 & $0 \%$ & 0.634 & $\mathrm{~F}$ & 0.074 & 0.136 \\
\hline \multicolumn{10}{|c|}{ Case vs non-BPH control } \\
\hline T vs $t$ & 6 & $1.54(1.20-1.98)$ & 3.42 & 0.001 & $18.8 \%$ & 0.291 & $\mathrm{~F}$ & 1.000 & 0.446 \\
\hline TT vs Tt & 6 & $1.41(1.06-1.87)$ & 2.39 & 0.017 & $0 \%$ & 0.503 & $\mathrm{~F}$ & 0.707 & 0.479 \\
\hline TT vs tt & 6 & $3.53(1.40-8.94)$ & 2.67 & 0.008 & $0 \%$ & 0.905 & $\mathrm{~F}$ & 0.734 & 0.606 \\
\hline TT vs $(T t / t t)$ & 6 & $1.51(1.15-1.98)$ & 2.93 & 0.003 & $9.6 \%$ & 0.355 & $\mathrm{~F}$ & 1.000 & 0.493 \\
\hline$(\mathrm{TT} / \mathrm{Tt}) \mathrm{vs} \mathrm{tt}$ & 6 & $3.15(1.25-7.94)$ & 2.43 & 0.015 & $0 \%$ & 0.933 & $\mathrm{~F}$ & 0.734 & 0.614 \\
\hline \multicolumn{10}{|c|}{ BPH vs non-BPH Control } \\
\hline T vs $t$ & $3^{\mathrm{a}}$ & $1.68(0.92-3.09)^{\mathrm{a}}$ & $1.68^{\mathrm{a}}$ & $0.093^{a}$ & $60.6 \%^{\mathrm{a}}$ & $0.079^{a}$ & $\mathrm{R}^{\mathrm{a}}$ & $1.000^{\mathrm{a}}$ & $0.438^{\mathrm{a}}$ \\
\hline TT vs Tt & $3^{\mathrm{a}}$ & $1.67(0.80-3.47)^{\mathrm{a}}$ & $1.36^{\mathrm{a}}$ & $0.173^{\mathrm{a}}$ & $62.9 \%^{\mathrm{a}}$ & $0.068^{\mathrm{a}}$ & $\mathrm{R}^{\mathrm{a}}$ & $1.000^{\mathrm{a}}$ & $0.423^{\mathrm{a}}$ \\
\hline TT vs tt & 4 & $2.86(0.91-8.99)$ & 1.80 & 0.073 & $0 \%$ & 0.897 & $\mathrm{~F}$ & 0.296 & 0.111 \\
\hline TT vs $(T t / t t)$ & $3^{\mathrm{a}}$ & $1.75(0.85-3.58)^{\mathrm{a}}$ & $1.52^{\mathrm{a}}$ & $0.128^{a}$ & $64.3 \%^{\mathrm{a}}$ & $0.06 \mathrm{I}^{\mathrm{a}}$ & $\mathrm{R}^{\mathrm{a}}$ & $1.000^{\mathrm{a}}$ & $0.436^{\mathrm{a}}$ \\
\hline$(\mathrm{TT} / \mathrm{Tt}) \mathrm{vs} \mathrm{tt}$ & 4 & $2.4 \mathrm{I}(0.77-7.54)$ & 1.52 & 0.129 & $0 \%$ & 0.853 & $\mathrm{~F}$ & 0.296 & 0.056 \\
\hline
\end{tabular}

Note: 'Liu et al's ${ }^{17}$ study was excluded.

Abbreviations: BPH, benign prostatic hyperplasia; F, fixed effects model; M, effect model of meta-analysis; OR, odds ratio; PCa, prostate cancer; R, random effects model; VDR, vitamin D receptor.

A

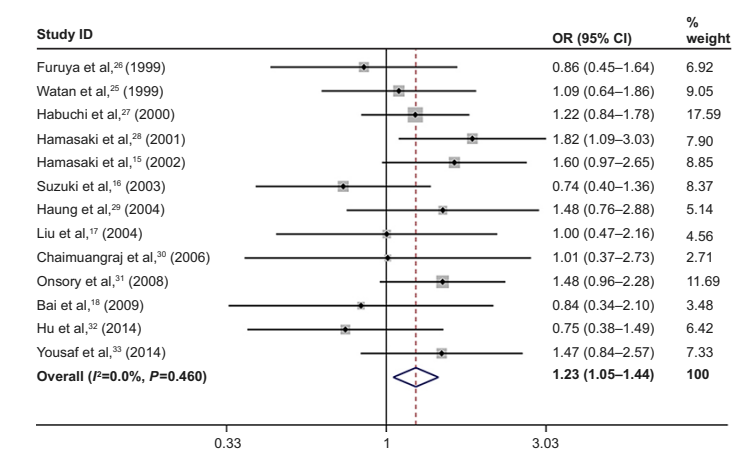

C

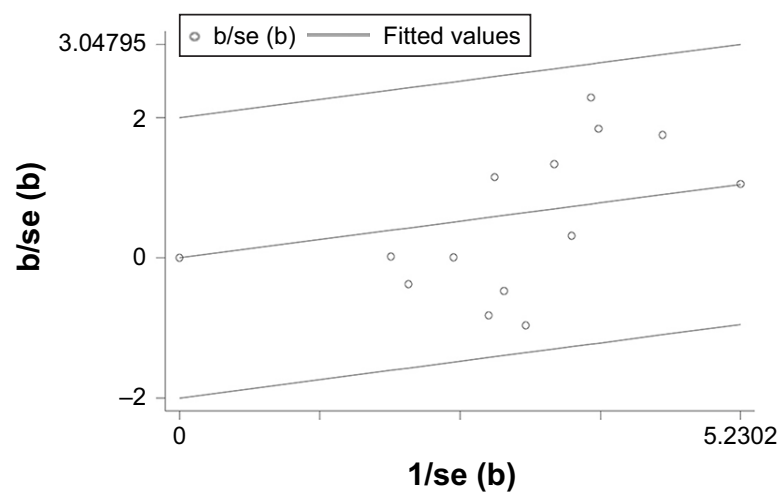

B

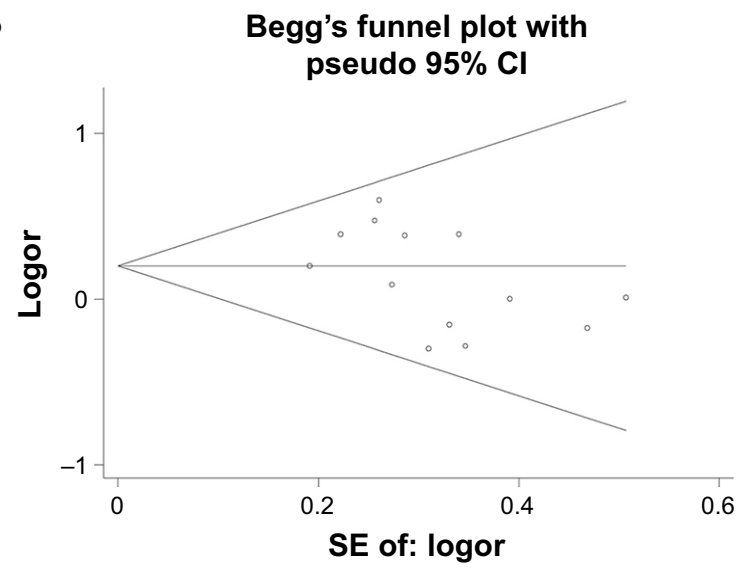

D

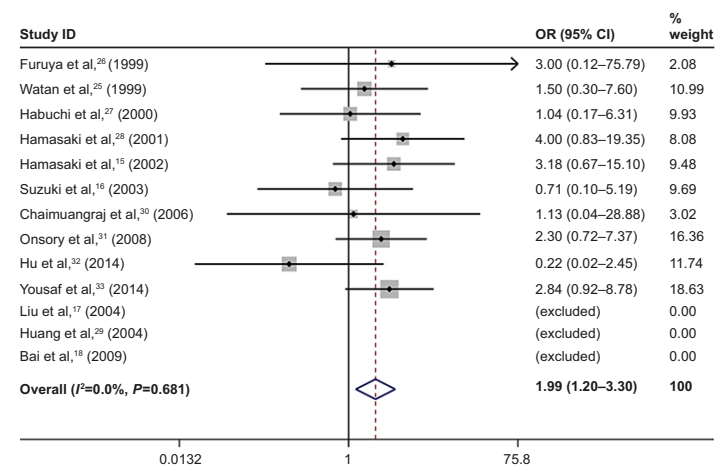

Figure 2 (Continued) 
$\mathbf{E}$

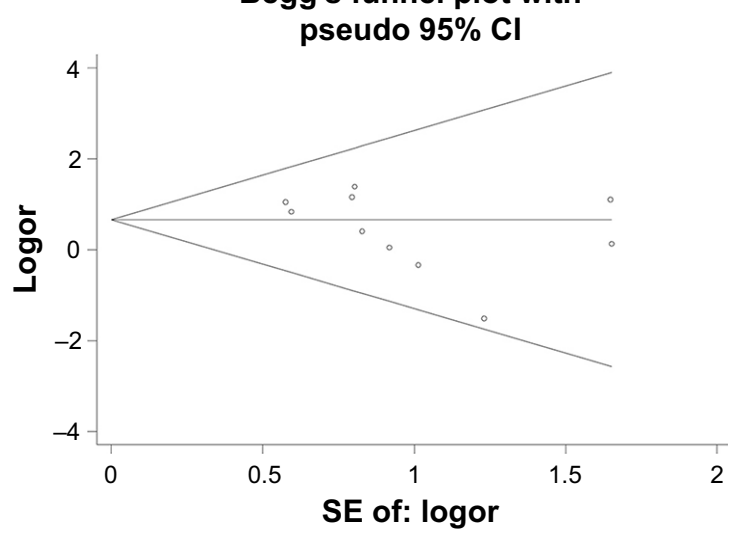

G

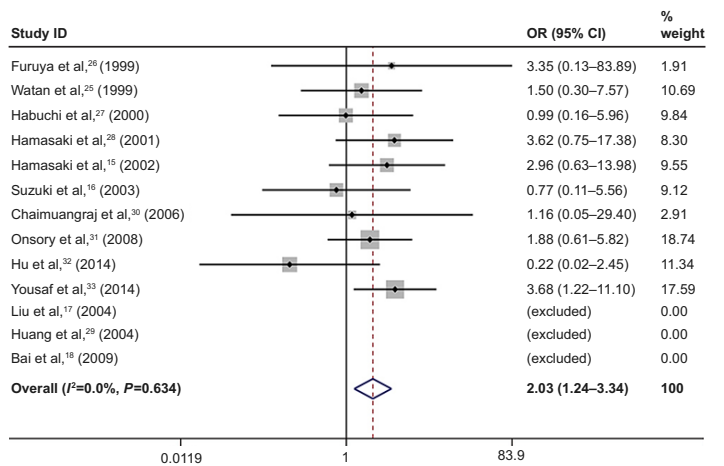

$\mathbf{F}$

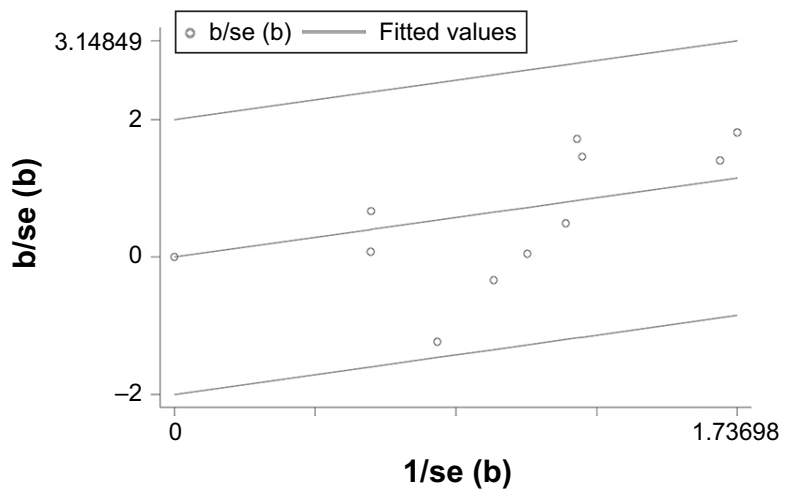

H

Begg's funnel plot with pseudo $95 \% \mathrm{Cl}$

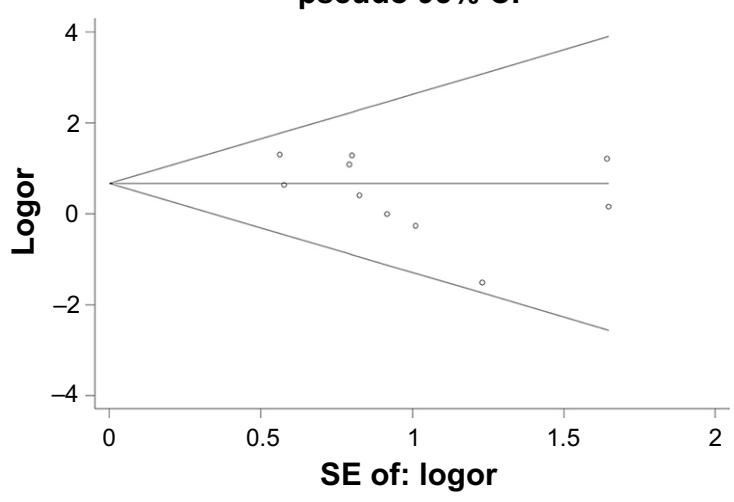

I

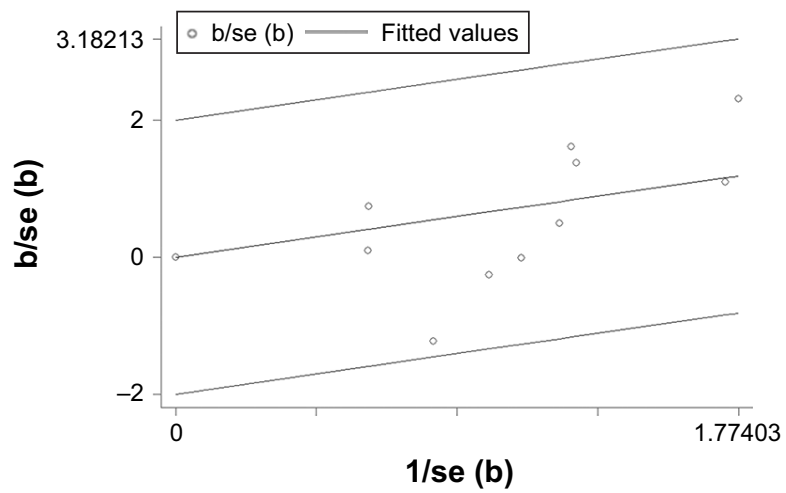

Figure 2 ORs, Begg's test and Galbraith blot test of PCa and VDR Taq I polymorphisms.

Note: T vs t $(\mathbf{A}-\mathbf{C})$, TT vs tt $(\mathbf{D}-\mathbf{F})$ and $(T T / T t)$ vs tt $(\mathbf{G}-\mathbf{I})$ were analyzed by fixed effects analysis.

Abbreviations: OR, odds ratio; PCa, prostate cancer; $\mathrm{SE}$, standard error; VDR, vitamin D receptor.

publication bias (Figure 4B and E), and the sensitivity analysis represented a good stability (Figure 4C and F).

\section{Association of VDR gene Taq I} polymorphism with PCa risk in different tumor and Gleason stages

According to the clinical pathological and Gleason stages, we performed a subgroup analysis to delineate the association of VDR gene Taq1 genetic polymorphism with PCa risk in more detail.

As shown in Table 4, in the clinical stage subgroup analysis, the pooled results revealed no significant association in local stage, but more frequency of $\mathrm{T}$ allele and TT genotype in the advanced group compared with control or local group (advanced group vs control group: $\mathrm{T}$ vs t: OR $[95 \% \mathrm{CI}]=1.62[1.16-2.25], P=0.004$; TT vs Tt: OR 
A
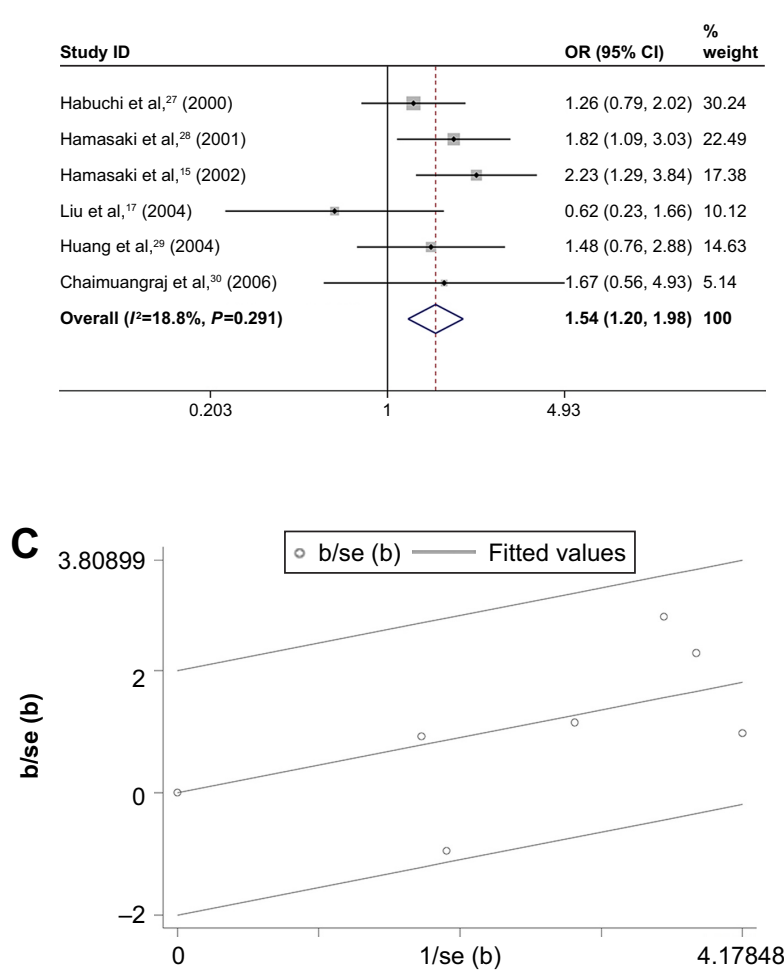

E
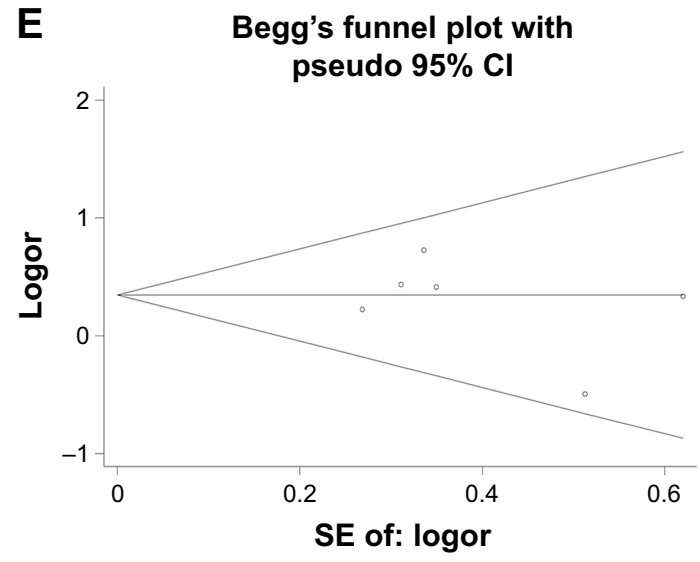

G

\begin{tabular}{|c|c|c|}
\hline Study ID & OR $(95 \% \mathrm{Cl})$ & $\begin{array}{l}\% \\
\text { weight }\end{array}$ \\
\hline Habuchi et al, ${ }_{27}^{27}(2000)$ & $1.83(0.25,13.22)$ & 26.56 \\
\hline Hamasaki et al, ${ }^{28}(2001)$ & $4.00(0.83,19.35)$ & 36.19 \\
\hline Hamasaki et al, ${ }^{15}(2002)$ & $4.66(0.91,23.91)$ & 28.32 \\
\hline Chaimuangraj et al, ${ }^{30}(2006)$ & $7_{3.14}(0.12,81.35)$ & 8.92 \\
\hline Liu et al, ${ }^{17}(2004)$ & (excluded) & 0.00 \\
\hline Huang et al, ${ }^{29}(2004)$ & (excluded) & 0.00 \\
\hline Overall $\left(I^{2}=0.0 \%, P=0.905\right)$ & $3.53(1.40,8.94)$ & 100 \\
\hline
\end{tabular}

B

\section{Begg's funnel plot with pseudo $95 \% \mathrm{Cl}$}

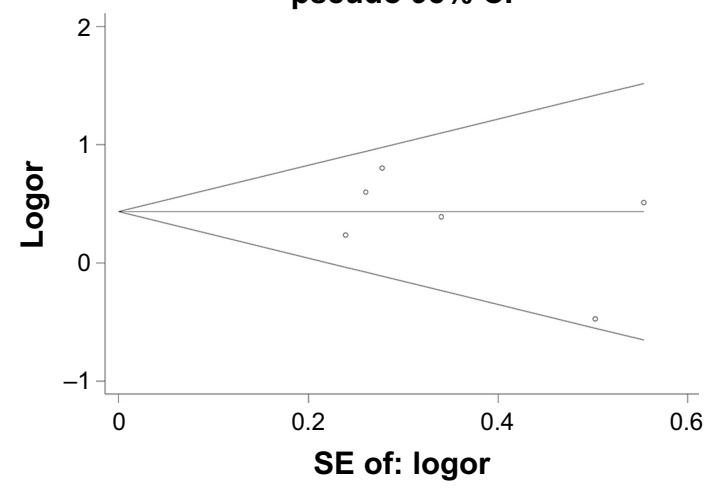

D

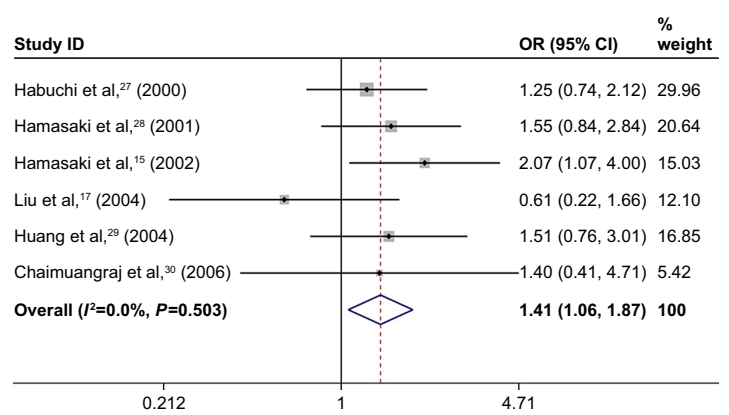

$\mathbf{F}$

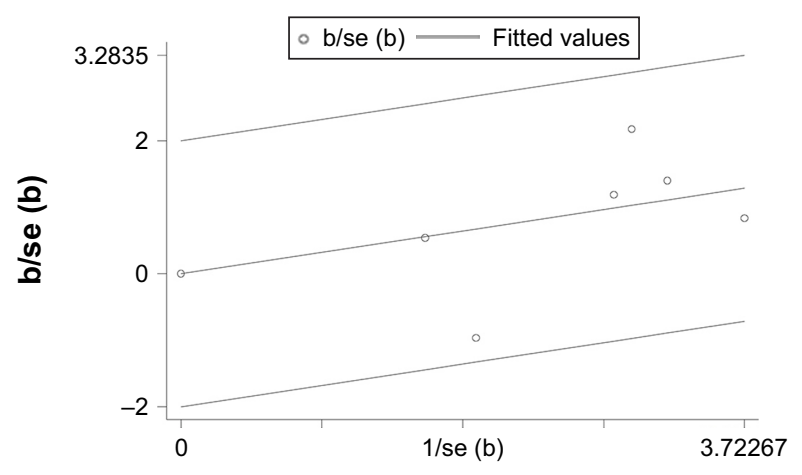

H Begg's funnel plot with
pseudo $95 \% \mathrm{Cl}$

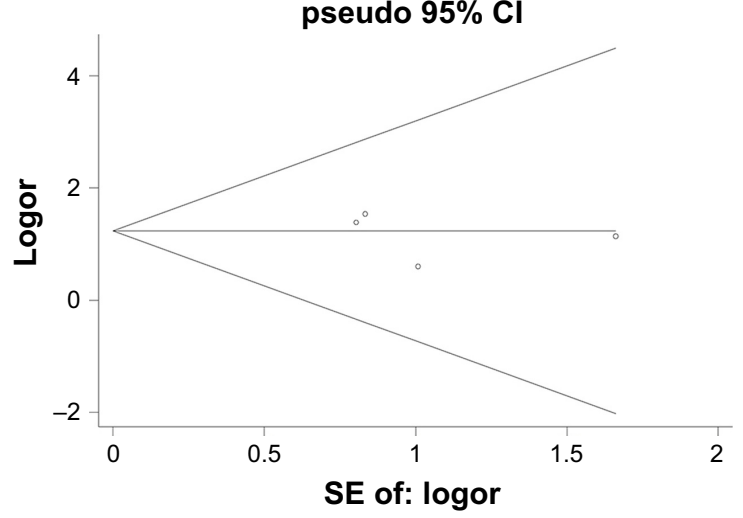

Figure 3 (Continued) 
I

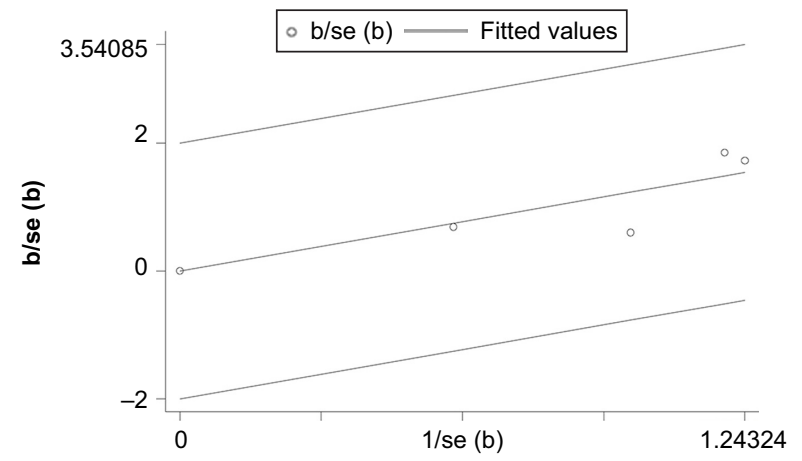

\section{$\mathbf{K}$}

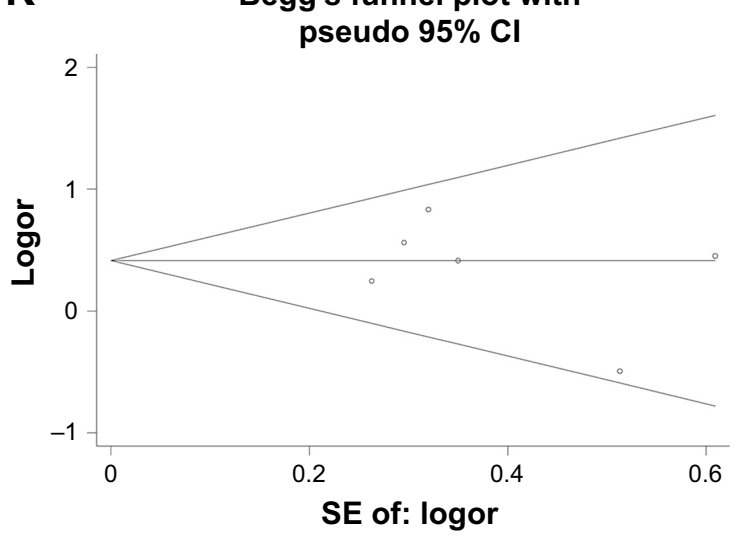

M

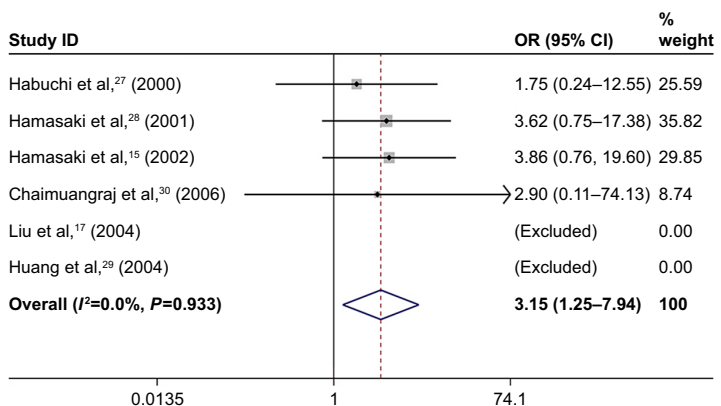

J

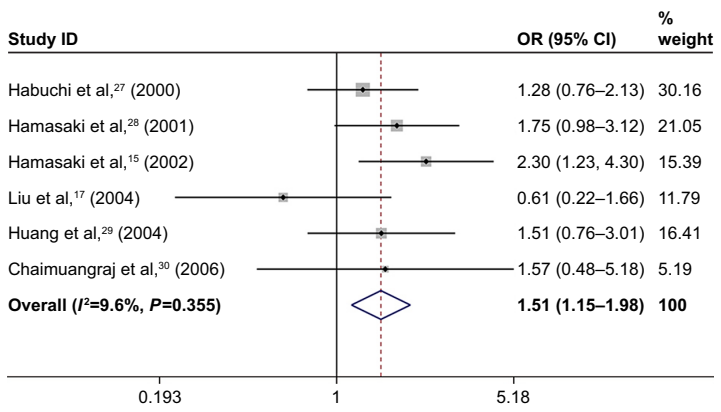

$\mathbf{L}$

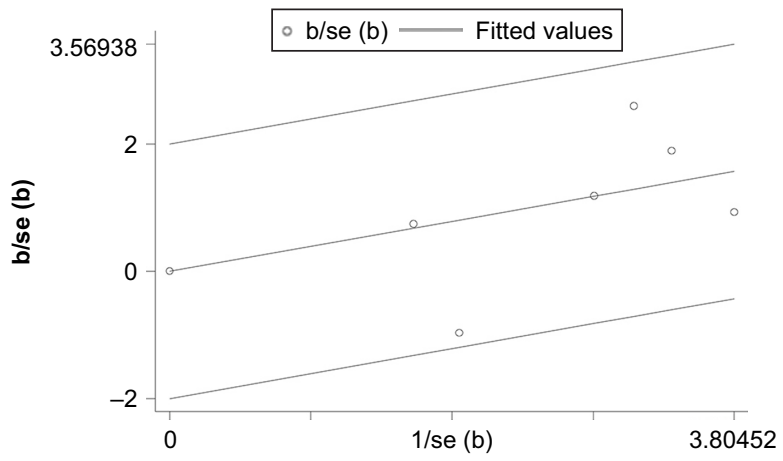

N Begg's funnel plot with pseudo $95 \% \mathrm{Cl}$

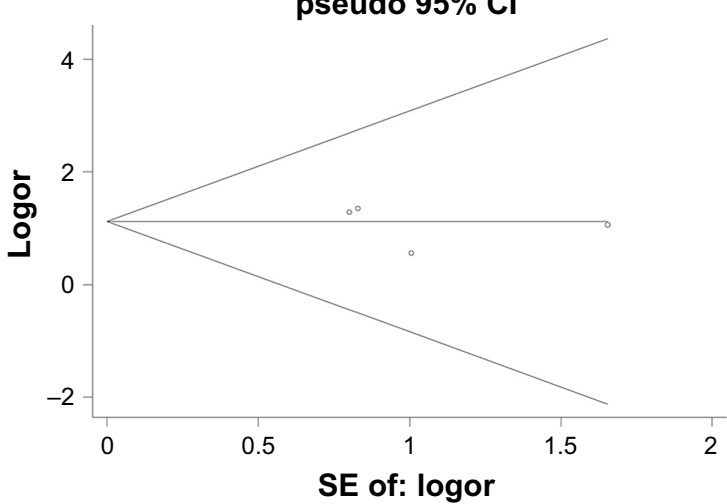

0

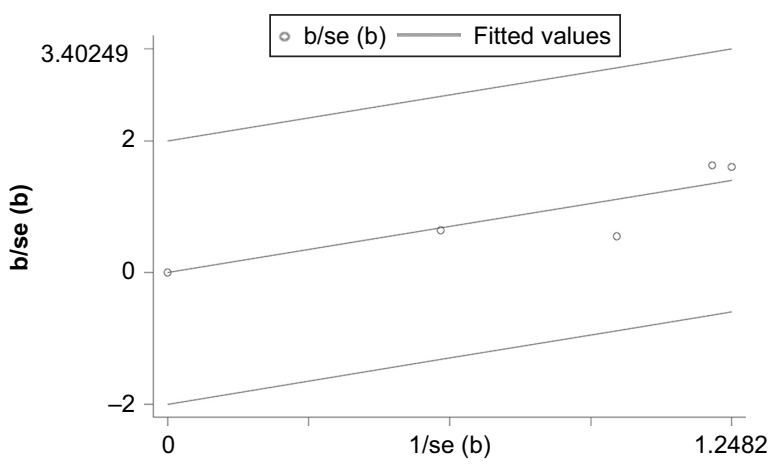

Figure 3 ORs, Begg's test and Galbraith blot test of case vs non-BPH as control.

Note: T vs t (A-C), TT vs Tt (D-F), TT vs tt (G-I), TT vs $(T t / t t)(\mathbf{J}-\mathbf{L})$ and $(T T / T t)$ vs tt $(\mathbf{M}-\mathbf{O})$ were analyzed by fixed models.

Abbreviations: $\mathrm{BPH}$, benign prostatic hyperplasia; OR, odds ratio. 
A

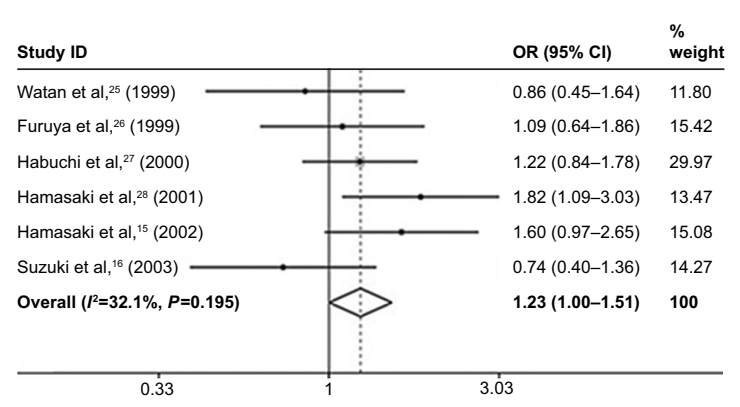

C

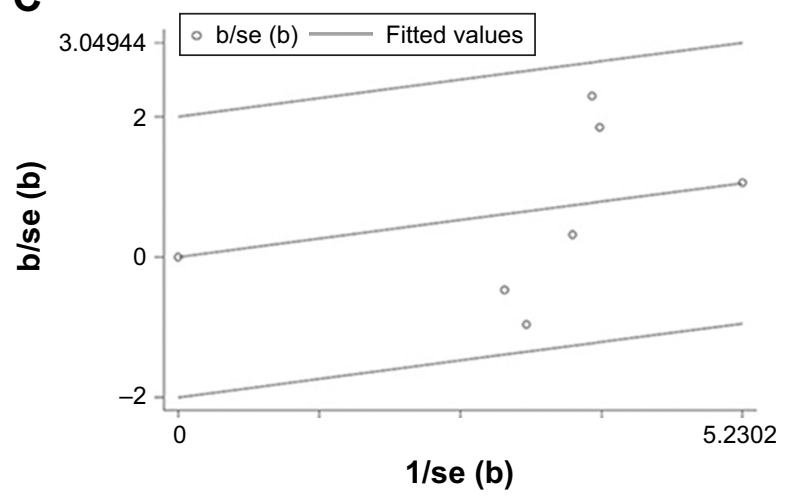

$\mathbf{E}$
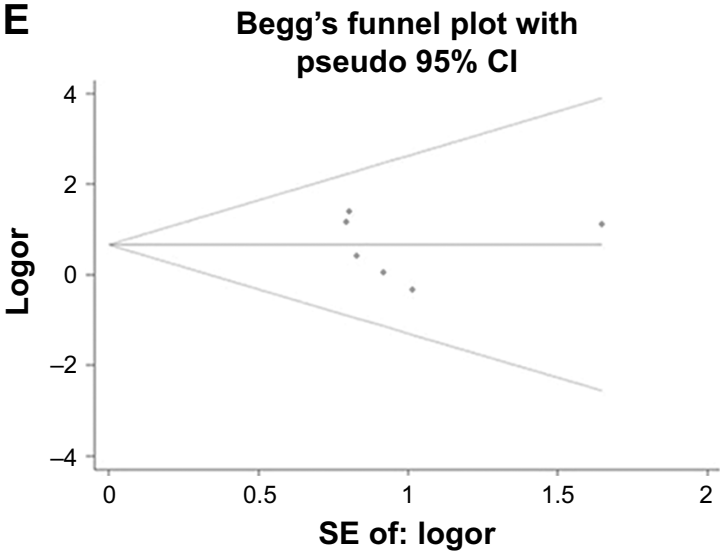

B

\section{Begg's funnel plot with} pseudo $95 \% \mathrm{Cl}$

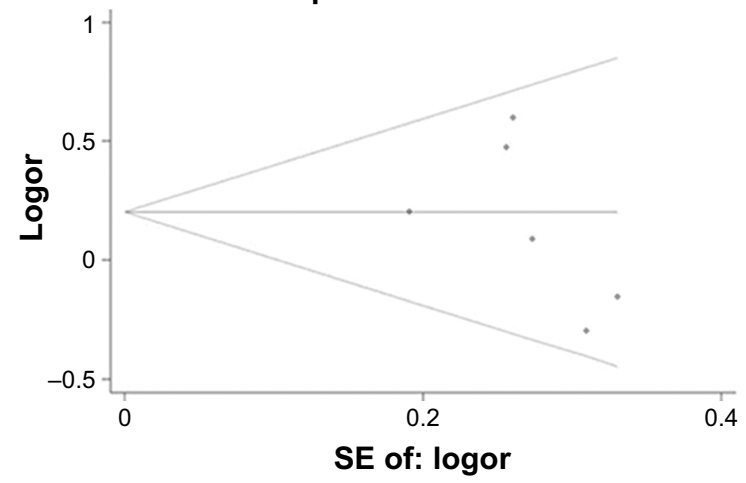

D

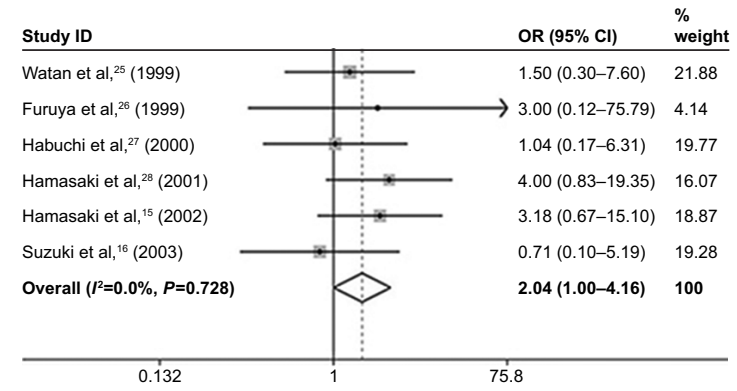

$\mathbf{F}$

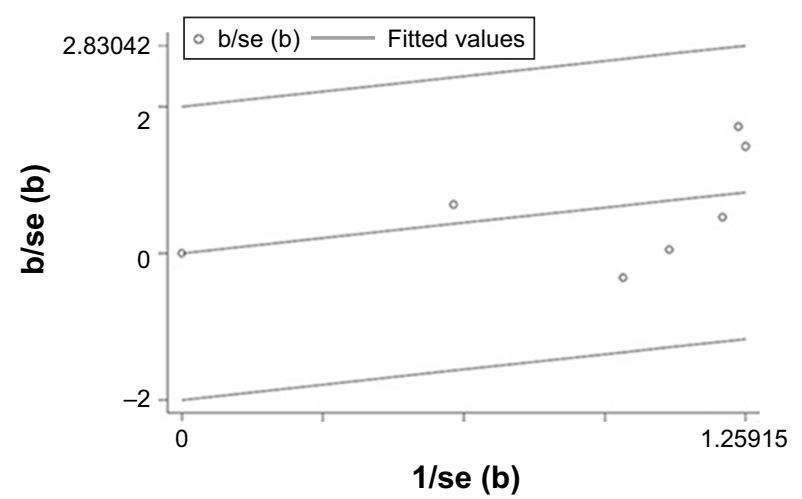

Figure 4 ORs, Begg's test and Galbraith blot test of PCa and VDR Taql polymorphisms in Japanese population.

Note: T vs t (A-C), TT vs tt (D-F) were analyzed by fixed effects analysis.

Abbreviations: OR, odds ratio; PCa, prostate cancer; VDR, vitamin D receptor.

[95\% CI] $=1.59[1.10-2.29], P=0.013 ;$ TT vs $[\mathrm{Tt} / \mathrm{tt}]:$ OR [95\% CI] $=1.80$ [1.25-2.61], $P=0.002$; advanced group vs local group: T vs t: OR $[95 \% \mathrm{CI}]=1.56[1.02-2.38], P=0.041$; TT vs Tt: OR $[95 \% \mathrm{CI}]=2.10[1.33-3.31], P=0.001$; TT vs $[\mathrm{Tt} / \mathrm{tt}]:$ OR $[95 \% \mathrm{CI}]=1.68[1.07-2.62], P=0.023$; Table 4 and Figure 5).

As shown in Table 5, in the Gleason subgroup analysis, the combined results showed no significant association between Gleason $<7$ vs control group, but the $\mathrm{T}$ allele and
TT genotype were significantly higher in Gleason $\geq 7$ group than control group or Gleason $<7$ (Table 5 and Figure 6).

\section{Sensitivity and heterogeneity analysis}

Sensitivity analysis was performed to test data stability by omitting one study at a time. The significance of all ORs was unchanged. A sensitivity analysis indicated that an independent study by Liu et a $1{ }^{17}$ was the principal reference for heterogeneity of TaqI polymorphism in the development of BPH. 
Table 3 Meta-analysis of the association of VDR gene Taql polymorphism with PCa risk in Asian different countries

\begin{tabular}{|c|c|c|c|c|c|c|c|c|c|}
\hline \multirow[t]{2}{*}{$\overline{\text { Gene }}$} & \multirow[t]{2}{*}{ Studies } & \multicolumn{3}{|c|}{ Test for overall effect } & \multicolumn{2}{|c|}{ Heterogeneity } & \multirow[t]{2}{*}{$\mathbf{M}$} & \multicolumn{2}{|c|}{ Publication bias } \\
\hline & & OR (95\% Cl) & Z-score & $P$-value & $\overline{I^{2}}$ & $P$-value & & $\begin{array}{l}\text { Begg's } \\
\text { test }\end{array}$ & $\begin{array}{l}\text { Egger's } \\
\text { test }\end{array}$ \\
\hline \multicolumn{10}{|l|}{ China } \\
\hline T vs t & 4 & $1.02(0.70-1.47)$ & 0.09 & 0.929 & $0 \%$ & 0.544 & $\mathrm{~F}$ & 0.734 & 0.646 \\
\hline TT vs Tt & 4 & $1.10(0.75-1.63)$ & 0.50 & 0.618 & $0 \%$ & 0.720 & $\mathrm{~F}$ & 0.308 & 0.093 \\
\hline TT vs tt & 4 & $0.22(0.02-2.45)$ & 1.23 & 0.218 & - & - & - & - & - \\
\hline TT vs (Tt/tt) & 4 & $1.06(0.72-1.56)$ & 0.30 & 0.762 & $0 \%$ & 0.638 & $\mathrm{~F}$ & 0.734 & 0.335 \\
\hline$(\mathrm{TT} / \mathrm{Tt}$ ) vs tt & 4 & $0.22(0.02-2.45)$ & 1.23 & 0.218 & - & - & - & - & - \\
\hline \multicolumn{10}{|l|}{ Japan } \\
\hline T vs t & 6 & $1.23(1.00-1.5 \mathrm{I})$ & 1.97 & 0.049 & $32.1 \%$ & 0.195 & $\mathrm{~F}$ & 0.26 & 0.394 \\
\hline TT vs Tt & 6 & $1.14(0.90-1.44)$ & 1.07 & 0.287 & $0.40 \%$ & 0.413 & $\mathrm{~F}$ & 0.26 & 0.190 \\
\hline TT vs tt & 6 & $2.04(1.00-4.16)$ & 1.96 & 0.048 & $0 \%$ & 0.728 & $\mathrm{~F}$ & 0.26 & 0.708 \\
\hline TT vs (Tt/tt) & 6 & $1.20(0.95-1.50)$ & 1.54 & 0.124 & $21.8 \%$ & 0.270 & $\mathrm{~F}$ & 0.26 & 0.287 \\
\hline (TT/Tt) vs tt & 6 & $1.97(0.97-4.02)$ & 1.87 & 0.061 & $0 \%$ & 0.777 & $\mathrm{~F}$ & 0.26 & 0.810 \\
\hline \multicolumn{10}{|l|}{ India } \\
\hline T vs t & I & $1.48(0.96-2.28)$ & 1.76 & 0.079 & - & - & - & - & - \\
\hline TT vs Tt & I & $1.53(0.86-2.74)$ & 1.45 & 0.147 & - & - & - & - & - \\
\hline TT vs tt & I & $2.30(0.72-7.37)$ & 1.40 & 0.160 & - & - & - & - & - \\
\hline TT vs (Tt/tt) & I & $1.62(0.93-2.83)$ & 1.69 & 0.090 & - & - & - & - & - \\
\hline (TT/Tt) vs tt & I & $1.88(0.61-5.82)$ & 1.09 & 0.274 & - & - & - & - & - \\
\hline \multicolumn{10}{|l|}{ Thailand } \\
\hline$T$ vs $t$ & I & I.0I (0.37-2.73) & 0.02 & 0.984 & - & - & - & - & - \\
\hline $\mathrm{TT}$ vs Tt & I & $0.87(0.30-2.55)$ & 0.25 & 0.800 & - & - & - & - & - \\
\hline TT vs tt & I & $1.13(0.05-28.9)$ & 0.08 & 0.939 & - & - & - & - & - \\
\hline TT vs (Tt/tt) & I & $0.89(0.31-2.59)$ & 0.21 & 0.831 & - & - & - & - & - \\
\hline (TT/Tt) vs tt & I & $1.16(0.05-29.4)$ & 0.09 & 0.927 & - & - & - & - & - \\
\hline \multicolumn{10}{|l|}{ Pakistan } \\
\hline T vs t & I & $1.47(0.84-2.57)$ & 1.34 & 0.180 & - & - & - & - & - \\
\hline TT vs Tt & I & $0.30(0.12-0.75)$ & 2.57 & 0.010 & - & - & - & - & - \\
\hline TT vs tt & I & $2.84(0.92-8.78)$ & 1.81 & 0.070 & - & - & - & - & - \\
\hline TT vs (Tt/tt) & I & $0.90(0.44-1.83)$ & 0.29 & 0.769 & - & - & - & - & - \\
\hline (TT/Tt) vs tt & I & $3.68(1.22-11.1)$ & 2.31 & 0.021 & - & - & - & - & - \\
\hline
\end{tabular}

Note: “-” indicates no data.

Abbreviations: F, fixed effects model; M, effect model of meta-analysis; OR, odds ratio; PCa, prostate cancer; VDR, vitamin D receptor.

Table 4 Meta-analysis of the association of VDR gene Taql polymorphism with PCa risk in different tumor stages

\begin{tabular}{|c|c|c|c|c|c|c|c|c|c|}
\hline \multirow[t]{2}{*}{ Gene } & \multirow[t]{2}{*}{ Studies } & \multicolumn{3}{|c|}{ Test for overall effect } & \multicolumn{2}{|c|}{ Heterogeneity } & \multirow[t]{2}{*}{$M$} & \multicolumn{2}{|c|}{ Publication bias } \\
\hline & & OR $(95 \% \mathrm{Cl})$ & Z-score & $P$-value & $I^{2}$ & $P$-value & & $\begin{array}{l}\text { Begg's } \\
\text { test }\end{array}$ & $\begin{array}{l}\text { Egger's } \\
\text { test }\end{array}$ \\
\hline \multicolumn{10}{|l|}{ Local vs control } \\
\hline T vs t & 5 & $1.02(0.74-1.40)$ & 0.10 & 0.924 & $0 \%$ & 0.721 & $\mathrm{~F}$ & 0.806 & 0.754 \\
\hline $\mathrm{TT}$ vs Tt & 6 & $0.77(0.55-1.09)$ & 1.47 & 0.141 & $0 \%$ & 0.727 & $\mathrm{~F}$ & 0.707 & 0.291 \\
\hline TT vs tt & 5 & $1.93(0.60-6.22)$ & 1.10 & 0.273 & $3.3 \%$ & 0.376 & $\mathrm{~F}$ & 0.734 & 0.437 \\
\hline TT vs $(T \mathrm{t} / \mathrm{tt})$ & 6 & $0.90(0.65-1.25)$ & 0.61 & 0.545 & $0 \%$ & 0.959 & $\mathrm{~F}$ & 0.707 & 0.580 \\
\hline$(\mathrm{TT} / \mathrm{Tt}$ ) vs tt & 6 & $1.77(0.63-4.93)$ & 1.08 & 0.278 & $0 \%$ & 0.521 & $\mathrm{~F}$ & 0.462 & 0.282 \\
\hline \multicolumn{10}{|c|}{ Advanced vs control } \\
\hline T vs t & 5 & $1.62(1.16-2.25)$ & 2.86 & 0.004 & $49 \%$ & 0.097 & $\mathrm{~F}$ & 0.462 & 0.762 \\
\hline TT vs Tt & 6 & $1.59(1.10-2.29)$ & 2.48 & 0.013 & $49.8 \%$ & 0.076 & $\mathrm{~F}$ & 1.000 & 0.901 \\
\hline TT vs tt & 5 & $2.18(0.80-5.90)$ & 1.53 & 0.126 & $0 \%$ & 0.949 & $\mathrm{~F}$ & 1.000 & 0.412 \\
\hline TT vs (Tt/tt) & 6 & $1.80(1.25-2.61)$ & 3.13 & 0.002 & $27.5 \%$ & 0.228 & $\mathrm{~F}$ & 0.133 & 0.111 \\
\hline$(\mathrm{TT} / \mathrm{Tt}) \mathrm{vs} \mathrm{tt}$ & 6 & $1.98(0.80-4.83)$ & 1.47 & 0.142 & $0 \%$ & 0.994 & $\mathrm{~F}$ & 0.806 & 0.691 \\
\hline \multicolumn{10}{|c|}{ Advanced vs local } \\
\hline T vs t & 5 & $1.56(1.02-2.38)$ & 2.04 & 0.041 & $0 \%$ & 0.798 & $\mathrm{~F}$ & 1.000 & 0.660 \\
\hline TT vs Tt & 6 & $2.10(1.33-3.31)$ & 3.20 & 0.001 & $19.5 \%$ & 0.286 & $\mathrm{~F}$ & 1.000 & 0.901 \\
\hline TT vs tt & 5 & $0.64(0.13-3.12)$ & 0.55 & 0.582 & $0 \%$ & 0.726 & $\mathrm{~F}$ & 0.296 & 0.028 \\
\hline TT vs (Tt/tt) & 6 & $1.68(1.07-2.62)$ & 2.27 & 0.023 & $0 \%$ & 0.557 & $\mathrm{~F}$ & 0.260 & 0.102 \\
\hline (TT/Tt) vs tt & 6 & $0.78(0.20-2.87)$ & 0.40 & 0.686 & $0 \%$ & 0.666 & $\mathrm{~F}$ & 1.000 & 0.387 \\
\hline
\end{tabular}

Abbreviations: F, fixed effects model; M, effect model of meta-analysis; OR, odds ratio; PCa, prostate cancer; VDR, vitamin D receptor. 
A

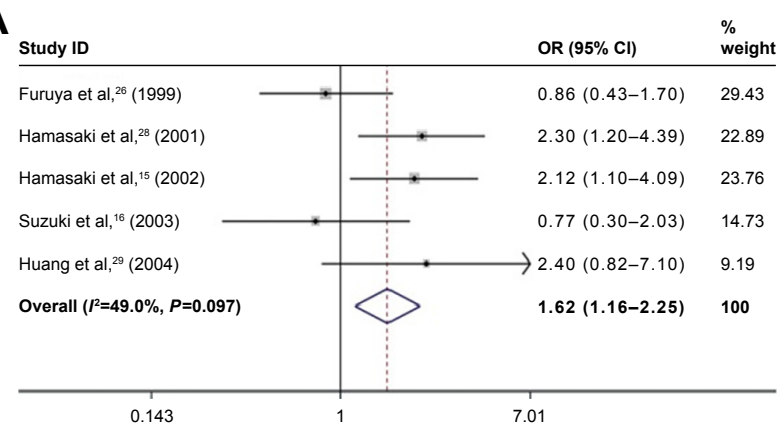

C Study ID Furuya et al, ${ }^{26}$ (1999) Hamasaki et al, ${ }^{28}$ (2001) Hamasaki et al, ${ }^{15}(2002)$ Suzuki et al, ${ }^{16}(2003)$ Huang et al, ${ }^{29}$ (2004) Liu et al, ${ }^{17}(2004)$ Overall $\left(I^{2}=49.8 \%, P=0.076\right)$

\begin{tabular}{llll} 
& & $1.59(1.10-2.29)$ & 100 \\
\hline 0.134 & & & 1 \\
\hline
\end{tabular}

E

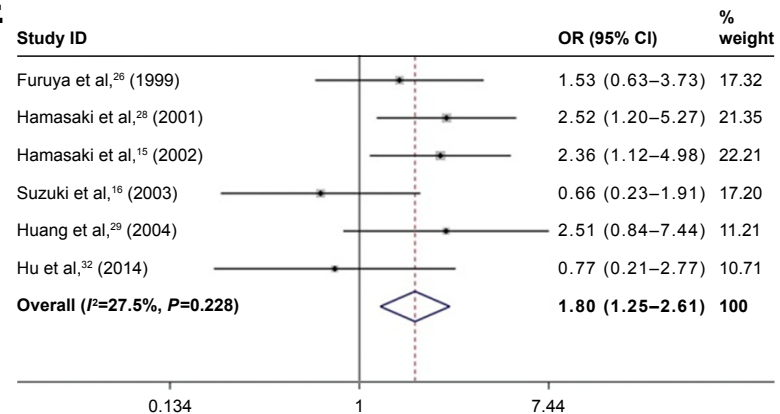

B

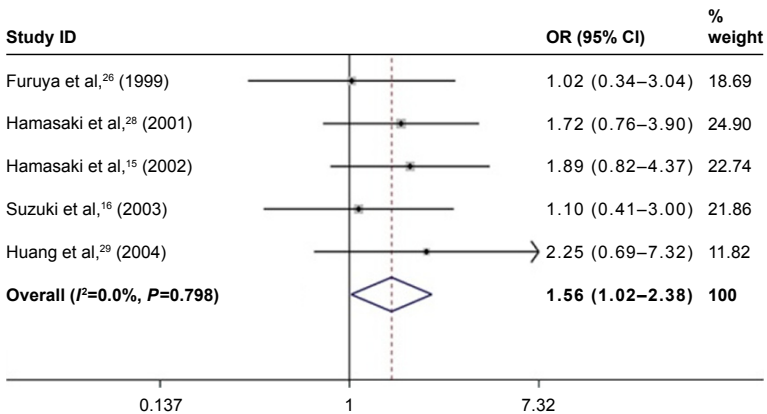

D Study ID OR $(95 \% \mathrm{Cl}) \quad \begin{aligned} & \% \\ & \text { weight }\end{aligned}$ Furuya et al, ${ }^{26}$ (1999) Hamasaki et al, ${ }^{28}$ (2001) Hamasaki et al, ${ }^{15}$ (2002) Suzuki et al, ${ }^{16}(2003)$ Huang et al, ${ }^{29}(2004)$ Liu et al, ${ }^{17}(2004)$ Overall $\left(I^{2}=19.5 \%, P=0.286\right)$ $1.03(0.30-3.59) \quad 18.68$ $2.80(1.08-7.26) \quad 19.03$ $3.25(1.21-8.72) \quad 16.55$ $0.83(0.27-2.56) \quad 25.22$ $2.33(0.70-7.78) \quad 14.53$ $4.86(1.11-21.25) 5.99$ $2.10(1.33-3.31) \quad 100$

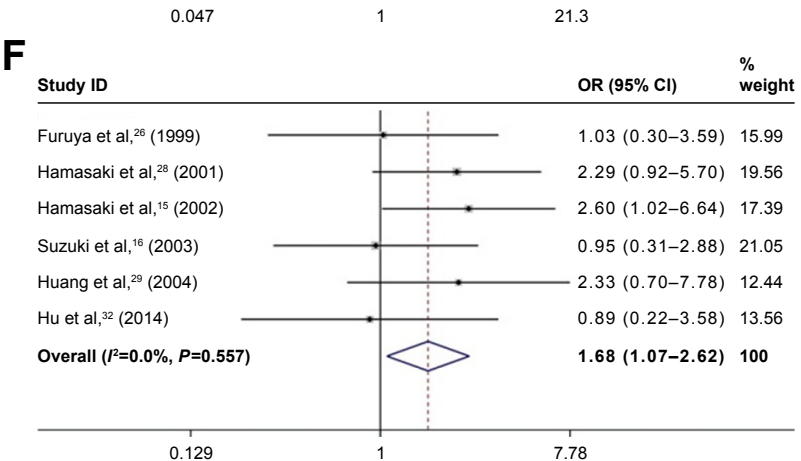

Figure 5 ORs of PCa associated with VDR Taql polymorphisms in different tumor stages.

Notes: T vs t (A, B), TT vs Tt (C, D) and TT vs (Tt/tt) (E, F) were analyzed by fixed effects analysis. (A, C, E: Advanced vs Control; B, D, F: Advanced vs Local). Abbreviations: OR, odds ratio; PCa, prostate cancer; VDR, vitamin D receptor.

Table 5 Meta-analysis of the association of VDR gene TaqI polymorphism with PCa risk in different Gleason stages

\begin{tabular}{|c|c|c|c|c|c|c|c|c|c|}
\hline \multirow[t]{2}{*}{ Gene } & \multirow[t]{2}{*}{ Studies } & \multicolumn{3}{|c|}{ Test for overall effect } & \multicolumn{2}{|c|}{ Heterogeneity } & \multirow[t]{2}{*}{ M } & \multicolumn{2}{|c|}{ Publication bias } \\
\hline & & OR $(95 \% \mathrm{Cl})$ & Z-score & $P$-value & $I^{2}$ & $P$-value & & $\begin{array}{l}\text { Begg's } \\
\text { test }\end{array}$ & $\begin{array}{l}\text { Egger's } \\
\text { test }\end{array}$ \\
\hline \multicolumn{10}{|c|}{ Gleason $<7$ vs control } \\
\hline T vs t & 4 & $1.31(0.96-1.78)$ & 1.68 & 0.093 & $0 \%$ & 0.617 & $\mathrm{~F}$ & 0.089 & 0.057 \\
\hline TT vs Tt & 5 & $1.14(0.81-1.62)$ & 0.76 & 0.449 & $0 \%$ & 0.927 & $\mathrm{~F}$ & 0.221 & 0.146 \\
\hline TT vs tt & 4 & $2.20(0.81-5.93)$ & 1.55 & 0.121 & $0 \%$ & 0.384 & $\mathrm{~F}$ & 0.296 & 0.081 \\
\hline TT vs $(T t / t t)$ & 5 & $1.18(0.85-1.62)$ & 0.99 & 0.322 & $0 \%$ & 0.717 & $\mathrm{~F}$ & 0.086 & 0.120 \\
\hline$(\mathrm{TT} / \mathrm{Tt}) \mathrm{vs} \mathrm{tt}$ & 4 & $2.10(0.78-5.66)$ & 1.47 & 0.141 & $0 \%$ & 0.397 & $\mathrm{~F}$ & 0.296 & 0.067 \\
\hline \multicolumn{10}{|c|}{ Gleason $\geq 7$ vs control } \\
\hline T vs t & $2^{\mathrm{a}}$ & $4.32(1.85-10.1)^{\mathrm{a}}$ & $3.39^{a}$ & $0.00 \mathrm{I}^{\mathrm{a}}$ & $0 \%^{\mathrm{a}}$ & $0.499^{a}$ & $\mathrm{~F}^{\mathrm{a}}$ & $1.000^{\mathrm{a}}$ & - \\
\hline TT vs Tt & $3^{\mathrm{a}}$ & $2.21(0.80-6.12)^{\mathrm{a}}$ & $1.52^{\mathrm{a}}$ & $0.128^{\mathrm{a}}$ & $58.7 \%^{\mathrm{a}}$ & $0.089^{\mathrm{a}}$ & $\mathrm{R}^{\mathrm{a}}$ & $0.296^{\mathrm{a}}$ & $0.066^{\mathrm{a}}$ \\
\hline TT vs tt & 3 & $2.89(0.5 \mathrm{I}-16.6)$ & 1.19 & 0.232 & $6.2 \%$ & 0.302 & $\mathrm{~F}$ & 1.000 & - \\
\hline TT vs (Tt/tt) & $3^{a}$ & $2.94(1.45-5.96)^{\mathrm{a}}$ & $2.98^{\mathrm{a}}$ & $0.003^{\mathrm{a}}$ & $45.5 \% \mathrm{a}$ & $0.159^{a}$ & $\mathrm{~F}^{\mathrm{a}}$ & $1.000^{\mathrm{a}}$ & $0.309^{a}$ \\
\hline$(\mathrm{TT} / \mathrm{Tt}$ ) vs tt & 3 & $2.61(0.45-15.2)$ & 1.06 & 0.288 & $0 \%$ & 0.402 & $\mathrm{~F}$ & 1.000 & - \\
\hline \multicolumn{10}{|c|}{ Gleason $\geq 7$ vs Gleason $<7$} \\
\hline T vs $\mathrm{t}$ & $2^{\mathrm{a}}$ & $3.69(1.51-9.01)^{\mathrm{a}}$ & $2.87^{a}$ & $0.004^{a}$ & $0 \%^{\mathrm{a}}$ & $0.712^{\mathrm{a}}$ & $\mathrm{F}^{\mathrm{a}}$ & $1.000^{\mathrm{a}}$ & - \\
\hline TT vs Tt & 4 & $1.78(0.98-3.25)$ & 1.89 & 0.059 & $48.3 \%$ & 0.122 & $\mathrm{~F}$ & 1.000 & 0.840 \\
\hline TT vs tt & 3 & $2.71(0.34-21.6)$ & 0.94 & 0.346 & $0 \%$ & 0.878 & $\mathrm{~F}$ & 1.000 & - \\
\hline TT vs $(T t / t t)$ & 4 & $1.87(1.04-3.37)$ & 2.08 & 0.037 & $47.4 \%$ & 0.127 & $\mathrm{~F}$ & 1.000 & 0.414 \\
\hline$(\mathrm{TT} / \mathrm{Tt} \mathrm{t}) \mathrm{vs} \mathrm{tt}$ & 3 & $2.63(0.32-21.7)$ & 0.90 & 0.369 & $0 \%$ & 0.974 & $\mathrm{~F}$ & 1.000 & - \\
\hline
\end{tabular}

Notes: ${ }^{2}$ Suzuki et al's ${ }^{16}$ study was excluded. “-” indicates no data.

Abbreviations: $F$, fixed effects model; $M$, effect model of meta-analysis; OR, odds ratio; $P C a$, prostate cancer; $R$, random effects model; VDR, vitamin $D$ receptor. 


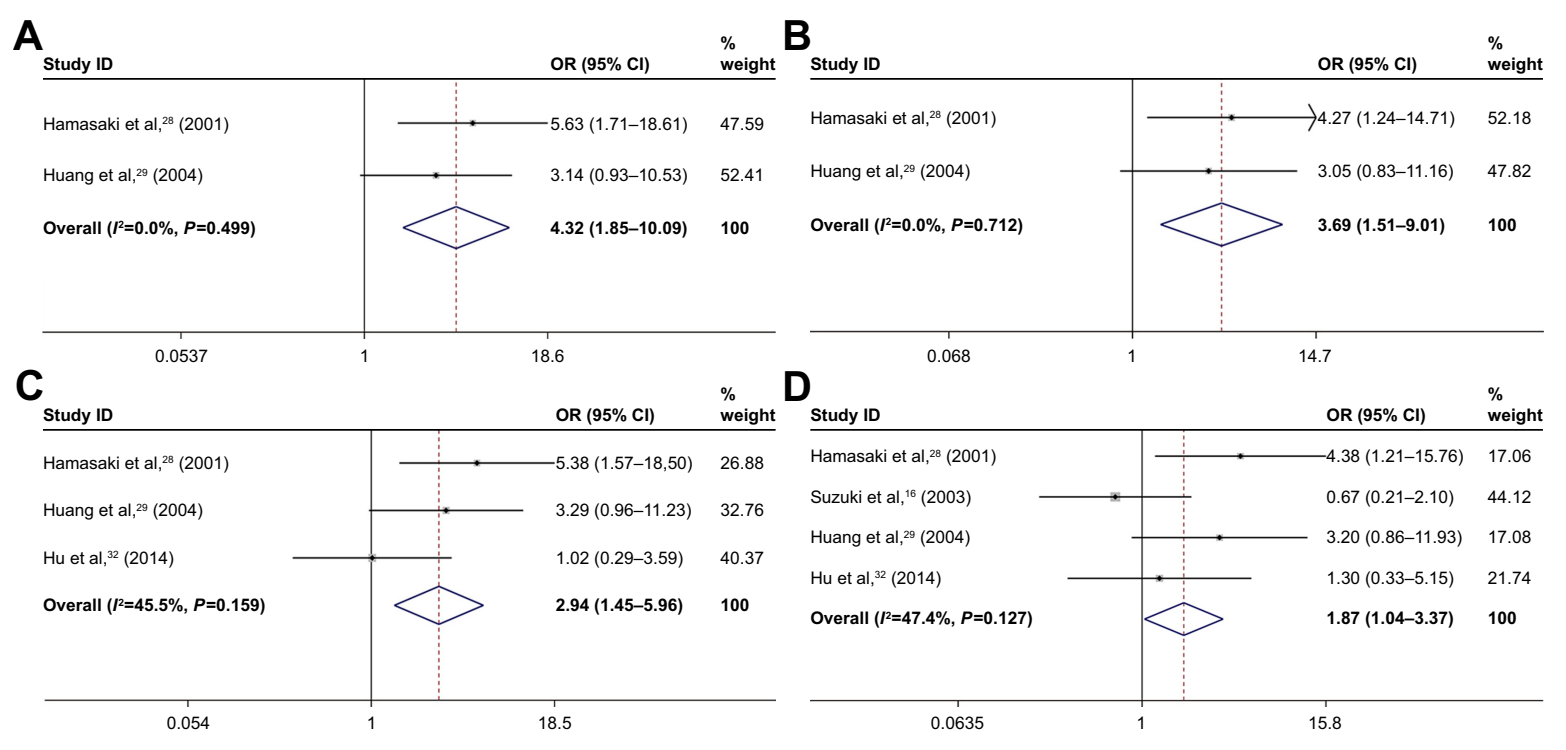

Figure 6 ORs of PCa associated with VDR Taql polymorphisms in different Gleason stages.

Notes: T vs t (A, B) and TT vs (Tt/tt) (C, D) were analyzed by fixed effects analysis. (A, C: Gleason $\geq 7$ vs Control; B, D: Gleason $\geq 7$ vs Gleason $<7$ ).

Abbreviations: OR, odds ratio; PCa, prostate cancer; VDR, vitamin D receptor.

After the exclusion of this study, we found it did not reduce the between-study heterogeneity obviously, and the outcome remained no statistical significance in the development of BPH (Table 2). In the subgroup analysis of Gleason $\geq 7$ vs Control or Gleason $\geq 7$ vs Gleason $<7$, the sensitivity analysis indicated that an independent study by Suzuki et $\mathrm{a}^{16}$ was the principal reference for heterogeneity of TaqI polymorphism in Gleason stage. After the exclusion of this study, the heterogeneity was effectively decreased or was eliminated (Table 5).

\section{Discussion}

Studies have shown that VDR Taq1 polymorphisms were associated with susceptibility to several carcinomas, such as breast cancer, oral cancer, and PCa. ${ }^{34-37}$ However, the association between VDR Taq1 genetic polymorphisms and PCa risk is still controversial, which may be caused by unscientific research or insufficient subjects and control numbers used in studies. In a study of Taylor et al, which included 96 cases of $\mathrm{PCa}$ and 162 non-BPH controls, they revealed that subjects carrying $\mathrm{tt}$ genotype have lower risk of $\mathrm{PCa}$ compared with $\mathrm{TT} / \mathrm{Tt}$ genotype $(\mathrm{OR}=0.32,95 \% \mathrm{CI}=0.15-0.75, P<0.01) .{ }^{8}$ Recently, similar results were also reported in studies by Liu et al, ${ }^{38}$ Guo et al, ${ }^{39}$ and Bonilla et $\mathrm{al}^{40}$, while 3 other studies ${ }^{12,41,42}$ showed different conclusions. To evaluate the association of VDR Taq1 genetic polymorphisms and susceptibility to PCa in Asian population, a comprehensive study of the previous research was performed by an updated systematic meta-analysis, which is the newest and most complete.
In the study of Fei et al,,${ }^{14}$ they mentioned that inclusion of population with BPH as control may bias the results. So we divided the subjects as 1), healthy, 2), BPH, and 3 ), case group. Combined analysis of 4 studies suggested no significant correlation between Taq1 VDR polymorphism and BPH risk. Besides, we conducted a subgroup analysis according to different Asian countries, which is an important biological factor that may affect the function of VDR by gene interaction. Interestingly, no association was shown except in the Japanese population. These inconsistent phenomena in different Asian countries may be a result of discrepant environmental conditions.

According to the different classification methods, TNM stage is based on the size of tumor diameter, lymph node metastasis, and distant metastasis staging, while Gleason stage is based on the degree of gland differentiation and tumor growth in the interstitial tissue. ${ }^{43}$ These 2 methods are independent, but could be complementary to assess the risk of cancer. Fei et al's study showed the genotypes TT and TT/Tt were significantly higher in the advanced PCa group compared with the control group, indicating that $\mathrm{T}$ allele is risker and easier to advanced stage,,${ }^{14}$ which was consistent with our results (Table 4 and Figure 5), while no statistically significant difference was shown according to Gleason staging. But in our study, we found a higher frequency of $\mathrm{T}$ allele gene and TT genotype in the Gleason $\geq 7$ stage PCa compared with Gleason $<7$ or Control, suggesting that $\mathrm{PCa}$ patients carrying $\mathrm{T}$ allele and TT genotype are more prone to entering an advanced stage (Table 5 and Figure 6). 
Moreover, there are several limitations of this metaanalysis. 1) Observational studies are susceptible to a wide variety of biases, such as genotyping errors, allelic heterogeneity, and selection bias; 2 ) in some studies, lack of clear explanation for the pathologic diagnostic results of subjects. The pathogenesis of sporadic PCa differs with hereditary PCa, which may lead to inevitable selection bias; 3) the conclusions of Gleason staging subgroup analyses might be limited because of a low statistical power of the small sample size; and 4) besides genetic polymorphisms, many factors, such as occupation, diet, smoking, age, and other confounding factors might contribute to the development of PCa.

\section{Conclusion}

Our results suggest that the VDR TaqI polymorphism might be associated with PCa risk in Asian male population, especially in the Japanese population. Moreover, PCa patients carrying $\mathrm{T}$ allele and TT genetype more easily progress to advanced stage. Therefore, these results indicate a high specificity and valuable biomarker to detect potential PCa in Asian population. Further steps should be taken to evaluate the gene-to-gene and gene-to-environment combined effect, and the total population and/or selected populations with different environmental background are urgently needed to conduct large scale multi-center epidemiological studies.

\section{Acknowledgments}

The excellent assistance of Kui Liu and Michael Hehir in the preparation of this article is greatly appreciated. This work was supported by Natural Science Foundation of Ningbo City (2016A610019).

\section{Disclosure}

The authors report no conflicts of interest in this work.

\section{References}

1. Siegel RL, Miller KD, Jemal A. Cancer statistics, 2016. CA Cancer J Clin. 2016;66(1):7-30.

2. Johns LE, Houlston RS. A systematic review and meta-analysis of familial prostate cancer risk. BJU Int. 2003;91(9):789-794.

3. Corder EH, Guess HA, Hulka BS, et al. Vitamin D and prostate cancer: a prediagnostic study with stored sera. Cancer Epidemiol Biomarkers Prev. 1993;2(5):467-472.

4. Peehl DM, Skowronski RJ, Leung GK, Wong ST, Stamey TA, Feldman D. Antiproliferative effects of 1,25-dihydroxyvitamin D3 on primary cultures of human prostatic cells. Cancer Res. 1994;54(3):805-810.

5. Liu Y, Li C, Chen P, et al. Polymorphisms in the vitamin D Receptor (VDR) and the risk of ovarian cancer: a meta-analysis. PLoS One. 2013; 8(6):e66716.

6. Bandera Merchan B, Morcillo S, Martin-Nunez G, Tinahones FJ, Macias-Gonzalez M. The role of vitamin D and VDR in carcinogenesis: Through epidemiology and basic sciences. J Steroid Biochem Mol Biol. 2017;167:203-218
7. Morrison NA, Qi JC, Tokita A, et al. Prediction of bone density from vitamin D receptor alleles. Nature. 1994;367(6460):284-287.

8. Taylor JA, Hirvonen A, Watson M, Pittman G, Mohler JL, Bell DA. Association of prostate cancer with vitamin D receptor gene polymorphism. Cancer Res. 1996;56(18):4108-4110.

9. Uitterlinden AG, Fang Y, Van Meurs JB, Pols HA, Van Leeuwen JP. Genetics and biology of vitamin D receptor polymorphisms. Gene. 2004; 338(2):143-156.

10. Li H, Stampfer MJ, Hollis JB, et al. A prospective study of plasma vitamin D metabolites, vitamin D receptor polymorphisms, and prostate cancer. PLoS Med. 2007;4(3):e103.

11. Ahn J, Peters U, Albanes D, et al; Prostate, Lung, Colorectal, and Ovarian Cancer Screening Trial Project Team. Serum vitamin D concentration and prostate cancer risk: a nested case-control study. J Natl Cancer Inst. 2008;100(11):796-804.

12. Ntais C, Polycarpou A, Ioannidis JP. Vitamin D receptor gene polymorphisms and risk of prostate cancer: a meta-analysis. Cancer Epidemiol Biomarkers Prev. 2003;12(12):1395-1402.

13. Berndt SI, Dodson JL, Huang WY, Nicodemus KK. A systematic review of vitamin D receptor gene polymorphisms and prostate cancer risk. J Urol. 2006;175(5):1613-1623.

14. Fei X, Liu N, Li H, Shen Y, Guo J, Wu Z. Polymorphisms of vitamin D receptor gene TaqI susceptibility of prostate cancer: a meta-analysis. Onco Targets Ther. 2016;9:1033-1045.

15. Hamasaki T, Inatomi H, Katoh T, Ikuyama T, Matsumoto T. Significance of vitamin $\mathrm{D}$ receptor gene polymorphism for risk and disease severity of prostate cancer and benign prostatic hyperplasia in Japanese. Urol Int. 2002;68(4):226-231.

16. Suzuki K, Matsui H, Ohtake N, et al. Vitamin D receptor gene polymorphism in familial prostate cancer in a Japanese population. Int J Urol. 2003;10(5):261-266.

17. Liu, J, Gu L, Ming L. Single nucleotide polymorphisms in the 3, region of vitamin D receptor gene and the genetic risk of prostate cancer in chinese population. Chin J Clin Rehabil. 2004;8:3429-3432.

18. Bai Y, Yu Y, Yu B, et al. Association of vitamin D receptor polymorphisms with the risk of prostate cancer in the Han population of Southern China. BMC Med Genet. 2009;10:125.

19. Ownby RL, Crocco E, Acevedo A, John V, Loewenstein D. Depression and risk for Alzheimer disease: systematic review, meta-analysis, and metaregression analysis. Arch Gen Psychiatry. 2006;63(5):530-538.

20. Higgins JP, Thompson SG. Quantifying heterogeneity in a metaanalysis. Stat Med. 2002;21(11):1539-1558.

21. Crippa A, Khudyakov P, Wang M, Orsini N, Spiegelman D. A new measure of between-studies heterogeneity in meta-analysis. Stat Med. 2016;35(21):3661-3675.

22. Nikolakopoulou A, Mavridis D, Salanti G. Demystifying fixed and random effects meta-analysis. Evid Based Ment Health. 2014;17(2):53-57.

23. Begg CB, Mazumdar M. Operating characteristics of a rank correlation test for publication bias. Biometrics. 1994;50(4):1088-1101.

24. Egger M, Davey Smith G, Schneider M, Minder C. Bias in meta-analysis detected by a simple, graphical test. BMJ. 1997;315(7109):629-634.

25. Watanabe M, Fukutome K, Murata M, et al. Significance of vitamin D receptor gene polymorphism for prostate cancer risk in Japanese. Anticancer Res. 1999;19(5C):4511-4514.

26. Furuya Y, Akakura K, Masai M, Ito H. Vitamin D receptor gene polymorphism in Japanese patients with prostate cancer. Endocr J. 1999; 46(3):467-470.

27. Habuchi T, Suzuki T, Sasaki R, et al. Association of vitamin D receptor gene polymorphism with prostate cancer and benign prostatic hyperplasia in a Japanese population. Cancer Res. 2000;60(2):305-308.

28. Hamasaki T, Inatomi H, Katoh T, Ikuyama T, Matsumoto T. Clinical and pathological significance of vitamin D receptor gene polymorphism for prostate cancer which is associated with a higher mortality in Japanese. Endocr J. 2001;48(5):543-549.

29. Huang SP, Chou YH, Wayne Chang WS, et al. Association between vitamin D receptor polymorphisms and prostate cancer risk in a Taiwanese population. Cancer Lett. 2004;207(1):69-77. 
30. Chaimuangraj S, Thammachoti R, Ongphiphadhanakul B, Thammavit W. Lack of association of VDR polymorphisms with Thai prostate cancer as compared with benign prostate hyperplasia and controls. Asian Pac J Cancer Prev. 2006;7(1):136-139.

31. Onsory K, Sobti RC, Al-Badran AI, et al. Hormone receptor-related gene polymorphisms and prostate cancer risk in North Indian population. Mol Cell Biochem. 2008;314(1-2):25-35.

32. Hu J, Qiu Z, Zhang L, Cui F. Kallikrein 3 and vitamin D receptor polymorphisms: potentials environmental risk factors for prostate cancer. Diagn Pathol. 2014;9:84.

33. Yousaf N, Afzal S, Hayat T, et al. Association of vitamin D receptor gene polymorphisms with prostate cancer risk in the Pakistani population. Asian Pac J Cancer Prev. 2014;15(22):10009-10013.

34. Abbas S, Nieters A, Linseisen J, et al. Vitamin D receptor gene polymorphisms and haplotypes and postmenopausal breast cancer risk. Breast Cancer Res. 2008;10(2):R31.

35. Tang C, Chen N, Wu M, Yuan H, Du Y. Fok1 polymorphism of vitamin D receptor gene contributes to breast cancer susceptibility: a meta-analysis. Breast Cancer Res Treat. 2009;117(2):391-399.

36. Mishra DK, Wu Y, Sarkissyan M, et al. Vitamin D receptor gene polymorphisms and prognosis of breast cancer among African-American and Hispanic women. PLoS One. 2013;8(3):e57967.
37. Bektas-Kayhan K, Unur M, Yaylim-Eraltan I, et al. Association of vitamin D receptor Taq I polymorphism and susceptibility to oral squamous cell carcinoma. In Vivo. 2010;24(5):755-759.

38. Liu D, Liu C, Ding B. Polymorphism of vitamin D receptor gene Taq and genetic susceptibility to prostate cancer in Asian man:a meta-analysis. J Mod Urol. 2011;3:222-226.

39. Guo YJ, Shi ZM, Liu JD, Lei N, Chen QH, Tang Y. Meta-analysis of the relation between the VDR gene TaqIpolymorphism and genetic susceptibility to prostate cancer in Asian populations. Asian Pac J Cancer Prev. 2012;13(9):4441-4444.

40. Bonilla C, Hooker S, Mason T, Bock CH, Kittles RA. Prostate cancer susceptibility Loci identified on chromosome 12 in African Americans. PLoS One. 2011;6(2):e16044.

41. Qi H, Xiaxi L, Jian S. Vitamin D receptor gene Taq I genetic Polymorphism in the prostate cancer of asian man:a meta-analysis. Chinese Journal of Gerontology. 2012;8:1571-1574.

42. Andersson P, Varenhorst E, Soderkvist P. Androgen receptor and vitamin D receptor gene polymorphisms and prostate cancer risk. Eur J Cancer. 2006;42(16):2833-2837.

43. Cooperberg MR. Implications of the new AUA guidelines on prostate cancer detection in the U.S. Curr Urol Rep. 2014;15(7):420.
OncoTargets and Therapy

\section{Publish your work in this journal}

OncoTargets and Therapy is an international, peer-reviewed, open access journal focusing on the pathological basis of all cancers, potential targets for therapy and treatment protocols employed to improve the management of cancer patients. The journal also focuses on the impact of management programs and new therapeutic agents and protocols on

\section{Dovepress}

patient perspectives such as quality of life, adherence and satisfaction The manuscript management system is completely online and includes a very quick and fair peer-review system, which is all easy to use. Visit http://www.dovepress.com/testimonials.php to read real quotes from published authors. 